\title{
Cardiovascular Magnetic Resonance assessment of 1st generation CoreValve and 2nd generation Lotus valves
}

\section{ABSTRACT}

Objectives: We sought to compare using serial CMR, the quantity of $A R$ and associated valve haemodynamics, following the first-generation CoreValve (Medtronic, Minneapolis, Minnesota) and the second-generation Lotus valve (Boston Scientific, Natick Massachusetts).

Background: Aortic regurgitation (AR) following Transcatheter Aortic Valve Replacement (TAVR) confers a worse prognosis and can be accurately quantified using cardiovascular magnetic resonance (CMR). Second generation valves have been specifically designed to reduce paravalvular AR and improve clinical outcomes.

Methods: Fifty-one patients ( $79.0 \pm 7.7$ years, $57 \%$ male) were recruited and imaged at three time points: immediately pre- and post-TAVR, and at 6 months.

Results: CMR-derived AR fraction immediately post-TAVR was greater in the CoreValve compared to Lotus group $(11.7 \pm 8.4$ vs. $4.3 \pm 3.4 \%, p=0.001)$, as was the frequency of $\geq$ moderate AR $(9 / 24(37.5 \%)$ vs. 0/27, $\mathrm{p}<0.001)$. However, at 6 months AR fraction had improved significantly in the CoreValve group such that the two valve designs were comparable (6.4 \pm 5.0 vs. $5.6 \pm 5.3 \%, p=0.623)$, with no patient in either group having $\geq$ moderate AR. The residual peak pressure gradient immediately following TAVR was significantly lower with CoreValve compared to Lotus $(14.1 \pm 5.6$ vs. $25.4 \pm 11.6 \mathrm{mmHg}$, $p=0.001)$, but again by 6 months the two valve designs were comparable $(16.5 \pm 9.4$ vs. $19.7 \pm 10.5 \mathrm{mmHg}, p=0.332)$. There was no difference in the degree of LV reverse remodelling between the two valves at 6 months.

Conclusion: Immediately post-TAVR, there was significantly less AR but a higher residual peak pressure gradient with the Lotus valve compared to CoreValve. However, at 6 months both devices had comparable valve haemodynamics and LV reverse 
remodelling.

KEYWORDS: Transcatheter Aortic Valve Replacement, Medtronic CoreValve, Boston Lotus Valve, Cardiovascular Magnetic Resonance, Aortic Regurgitation, Reverse Remodelling

Word count: 4,688 (including references)

\section{Abbreviations list}

AR:

aortic regurgitation

AS:

aortic stenosis

CMR:

cardiovascular magnetic resonance

IQR:

interquartile range

LV:

left ventricle

LVEDP:

left ventricular end diastolic pressure

LVEF

left ventricular ejection fraction

MDCT: multi detector computed tomography

TAVR: $\quad$ transcatheter aortic valve replacement

VARC: $\quad$ valve academic research consortium

VENC: $\quad$ velocity encoded gradient echo imaging 


\section{INTRODUCTION}

Transcatheter Aortic Valve Replacement (TAVR) device design has evolved in an attempt to improve both device success rates and clinical outcomes ${ }^{1}$. However, aortic regurgitation (AR) is seen in up to $80 \%$ of patients following TAVR, affecting both the balloon-expandable and the self-expanding designs ${ }^{2}$. This typically reflects incomplete circumferential apposition between the circular prosthesis and the oval-shaped aortic annulus ${ }^{3}$ and is often compounded by extensive calcification, under-expansion of the TAVR prosthesis or malposition ${ }^{4}$. Clinical trials and registry data have consistently shown that moderate or more paravalvular AR following TAVR is associated with reduced survival at short- and long-term term follow-up with all valve types ${ }^{5-7}$.

Cardiovascular magnetic resonance (CMR) is the reference modality for assessing LV mass, volumes and function. In addition, CMR permits full volumetric quantitation of AR that is highly accurate and reproducible 8.9 independent of the number or eccentricity of regurgitant jets ${ }^{10}$, and unlike echocardiography, is not limited by TAVR prosthesis or calcification artefact ${ }^{3}$. CMR has lower intra-observer and inter-observer variability than echocardiography 11,12 and thus is more suited to serial measurements. Compared with CMR, echocardiography underestimates AR following TAVR ${ }^{8,13}, 14$ and thus CMR offers a potentially superior prognostic assessment of the post-TAVR patient ${ }^{14}$.

Little is known about how AR evolves over time, especially with different valve designs, and how this might impact on LV reverse remodelling. Previous studies have indicated a reduction in AR over time with both the CoreValve and Edwards Sapien valve, but these data have been hampered by the limitations of transthoracic echocardiography in the assessment of paravalvular AR. The Boston Scientific Lotus valve has a unique adaptive seal specifically designed to minimise post-TAVR aortic regurgitation (AR) (8), that has proven to be both safe and effective in the REPRISE I ${ }^{15}$ and REPRISE II studies ${ }^{16}$. The aim of this study was to accurately quantify, using serial CMR, the degree of AR over 
time following TAVR using the first generation self-expanding Medtronic CoreValve ${ }^{17-21}$, and the second generation Boston Scientific Lotus valve, and to determine whether differences in aortic valve regurgitation and haemodynamics impact LV reverse remodelling.

\section{METHODS}

Study population

This non-randomised study prospectively recruited 59 patients with severe tri-leaflet degenerative AS who were referred for TAVR at the Leeds Teaching Hospitals NHS Trust, UK, between March 2013 and May 2015. Severe AS was classified by echocardiography as an aortic valve area of $\leq 1.0 \mathrm{~cm}^{2}$ or peak velocity $>4 \mathrm{~m} / \mathrm{s}$. Decision for TAVR in all cases was taken by a multidisciplinary heart team in accordance with international guidance ${ }^{22}$. In the initial part of the study period only the CoreValve device was available. Subsequently, device selection was made by the TAVI Heart Team according to individual specific patient anatomy and clinical implantation indications. Exclusion criteria included any contraindication to CMR as well as patients with a known bicuspid aortic valve, aortopathy or previous aortic or mitral prostheses. The study was approved by the national research ethics committee, complied with the Declaration of Helsinki and all patients provided written informed consent.

Transcatheter Aortic Valve Replacement

TAVR was performed using either a first generation CoreValve system (Medtronic, Minneapolis, Minnesota, USA) or the Lotus ${ }^{\mathrm{TM}}$ Aortic Valve system (Boston Scientific Corporation, Natick, MA, USA) employing standard techniques as previously described for both vendors ${ }^{23}{ }^{24}$. All patients underwent contrast-enhanced multi-detector computed tomography to assist annular sizing and to assess aortic calcification prior to TAVR. Percutaneous femoral artery access was the default approach, performed under either 
general anaesthesia or conscious sedation depending on patient suitability. CMR Protocol

For each individual patient identical pre- and post-TAVR, and 6-month post-operative scans were performed at 1.5T (Intera or Ingenia, Phillips Healthcare, Best, Netherlands) as previously described (Figure 1) ${ }^{25}$.26 $^{26}$ In brief, multi-slice, multi-phase cine imaging was performed using a standard steady-state free procession pulse sequence in the short axis $(8 / 0 \mathrm{~mm}, 30$ phases, typical field of view (FOV) $340 \mathrm{~mm})$ to cover the entire left and right ventricle. For flow measurements, through-plane velocity encoded (VENC) phase contrast imaging was performed perpendicular to the aortic valve jet at the aortic sinotubular junction, at the upper margin of the stent holding the TAVR prosthesis (VENC $200-500 \mathrm{~cm} / \mathrm{s}$, retrospective gating, slice thickness $6 \mathrm{~mm}, 40$ phases, FOV $340 \mathrm{~mm}$ ). This position for imaging has been previously described and validated ${ }^{9}$. If significant turbulence or aliasing was seen in the velocity image, the acquisition was repeated a few millimetres further away from the valve, and/or with a higher-velocity window. In patients with AF, the use of multiple acquisitions and averaging of values, and the application of arrhythmia rejection (in which data points acquired from excessively long or short heart beats are rejected and reacquired) were employed where feasible.

CMR Image Analysis

Image analysis was performed in a blinded fashion, off-line using commercially available software (CVI42, Circle Cardiovascular Imaging, Calgary, Alberta, Canada) by two experienced observers. For LV mass and volumes, standard criteria were employed to delineate endocardial and epicardial borders at end-diastole and end-systole and values obtained were indexed to body surface area as previously described ${ }^{25}$. Papillary muscles were included within the LV cavity for the purpose of analysis and excluded from the LV mass. Aortic flow was quantified using cross-sectional phase contrast images with 
contouring of the aortic lumen to provide a peak forward flow velocity $(\mathrm{m} / \mathrm{s})$, forward flow volume $(\mathrm{ml})$, backward flow volume $(\mathrm{ml})$ for the calculation of trans-valvular pressure gradient (Bernoulli equation) and regurgitant fraction (RF,\%). Images were excluded from analysis if artefacts from the TAVR were present on images. Aortic regurgitation was classified as regurgitant fraction of none/trivial $\leq 5 \%$, mild $6-15 \%$, moderate $16-25 \%$, moderate-severe $26-48 \%$, and severe $>48 \%$ in line with standard grading criteria ${ }^{27}$. Intraobserver (12 random data sets 6 months apart) and inter-observer (12 data sets) agreement was assessed using the intra-class correlation coefficient.

Sample Size and Statistical Analysis

Based on published data ${ }^{28} 20$ patients per group were required to detect a $10 \mathrm{ml}$ change in LVEDV or $10 \mathrm{~g}$ difference in LV mass regression between the two treatments $(90 \%$ power and an alpha error of 0.05$)$. A sample size of 16 patients per treatment group was required to adequately power a two-sample comparison of mean aortic regurgitant fraction (again at $90 \%$ power and an alpha error of 0.05 ). Continuous variables are presented as mean $\pm S D$. Normality was determined by the Shapiro-Wilk test. Frequencies are reported as number (\%). The Student t test and Wilcoxon signed rank test were used to compare continuous variables as appropriate, and X2 or Fisher's exact test for categorical comparisons. All statistical analyses were performed using the PASW software package (V.21.0 SPSS, IBM, Chicago, Illinois, USA) with a two-sided significance level of $p<0.05$ was considered statistically significant.

\section{RESULTS}

Patient population

A total of 51 patients (24 CoreValve and 27 Lotus valve) underwent both the pre-operative (median 1 day pre-procedure, IQR 14 days) and immediate post-TAVR (median 4 days, IQR 4 days) CMR scans with 44 of these (19 CoreValve and 25 Lotus) finally completing 
6-month post-TAVR scans. Reasons for non-completion of the CMR protocol were varied and are depicted in Figure 2. The final analysis population $(n=44)$ was not different from the whole population $(n=51)$ studied pre-TAVR (comparable in age $(p=0.871)$, EuroSCORE II $(p=0.724)$ and STS predicted operative mortality $(p=0.736))$. Baseline characteristics of the final study population are reported in Table 1.

Measurement Variability

Calculation of intra-class correlation coefficients indicated good intra- and inter-observer reproducibility of CMR measurements respectively: LVEDV (0.984, 0.989), LV mass $(0.978,0.985)$, LVEF $(0.982,0.970)$, peak aortic TAVR gradient $(1.000,0.963)$ and aortic regurgitant fraction post-TAVR $(0.987,0.986)$.

Procedural data

All of the Lotus valves were implanted via the femoral artery, as were the majority of CoreValves (67\% femoral, $29 \%$ subclavian, $4 \%$ direct aortic). The size and frequency of device replacement is detailed in Table 2. Invasive resting trans-aortic pressure gradients were equivalent between the two groups, in keeping with baseline imaging. The implant procedure for a Lotus valve involved significantly longer fluoroscopy times, despite a significantly greater proportion of CoreValve TAVR receiving post-dilatation $(0 \% \mathrm{vs.} 28 \%$, $\mathrm{p}=0.003$ ). Equivalent volumes of contrast were used for each TAVR device (Table 2). VARC-defined device success ${ }^{29}$ was achieved in $94 \%$ of the Lotus cohort and $63 \%$ of the CoreValve cohort $(p=0.004)$ at the immediate post-TAVR time point. The components of this measure were the absence of procedural mortality (94\% vs. $96 \%, p=0.177)$, a mean gradient across the TAVR prosthesis of $<20 \mathrm{mmHg}(100 \%$ vs. $100 \%, p=0.999)$, correct positioning of a single TAVR prosthesis (100\% vs. $96 \%, p=0.290)$, and no more than mild aortic regurgitation ( $100 \%$ vs. $63 \%, p=0.001)$ in the Lotus and CoreValve groups respectively. However, at the 6 month time-point, VARC defined success was 
equivalent between the two iterations; 91\% Boston Lotus Vs. 89\% for Medtronic CoreValve $(p=0.827)$. The absolute rate of new pacemaker insertion was similar between the 2 groups (22\% Lotus vs. 15\% CoreValve, $p=0.424)$.

Haemodynamics

The severity of pre-operative aortic valve stenosis was similar between the Lotus and CoreValve groups (Table 1). Systolic blood pressures (an important measure of LV afterload) remained comparable between the CoreValve and Lotus group both immediately $(132 \pm 23$ vs. $134 \pm 22 \mathrm{mmHg}, \mathrm{p}=0.784)$ and at 6 months $(141 \pm 25$ vs. $127 \pm 16 \mathrm{mmHg}$ respectively, $\mathrm{p}=0.161)$. Immediately post-TAVR, a significant reduction in peak aortic pressure gradient was observed in both Lotus (94.3 \pm 28.7 vs. $25.4 \pm 11.6 \mathrm{mmHg}, \mathrm{p}<0.001)$ and CoreValve $(88.5 \pm 27.4$ vs. $14.1 \pm 5.6 \mathrm{mmHg}, \mathrm{p}<0.001)$ groups. However, the residual peak pressure gradient measured by CMR immediately following Lotus valve replacement was significantly higher than that following CoreValve (25.4 \pm 11.6 vs. $14.1 \pm 5.6 \mathrm{mmHg}, \mathrm{p}=0.001)$. At 6 months post-TAVR, the peak pressure gradient of the CoreValve remained unchanged from the immediate post-TAVR time point (16.5 \pm 9.4 vs. $15.0 \pm 5.5 \mathrm{mmHg}$ respectively, $p=0.457)$; however a significant reduction was observed in the Lotus group (25.8 \pm 12.1 vs. $19.7 \pm 10.5 \mathrm{mmHg}, p=0.022)$ (Figure $5 \mathrm{~A})$. As such, in comparison between CoreValve and Lotus patients, the residual peak pressure gradient at 6 months was equivalent $(16.5 \pm 9.4$ vs.19.7 $\pm 10.5 \mathrm{mmHg}, \mathrm{p}=0.332)$ (Table 3). Immediately post-TAVR, the aortic regurgitant fraction was significantly greater in the CoreValve group ( $11.7 \pm 8.4$ vs. $4.3 \pm 3.4 \%, p=0.001)$. Similarly, the proportion of patients with $\geq$ moderate AR was significantly higher with CoreValve than Lotus (9/24 (37.5\%) vs. $0 / 27(0 \%), p<0.001$ ) (Figure 3). Between the immediate and 6 month scans, the aortic regurgitant fraction in the Lotus group remained unchanged $(4.0 \pm 3.5$ vs. $5.6 \pm 5.3 \%$, $\mathrm{p}=0.267$ ). However, a significant reduction was observed in the CoreValve patients 
(11.7 \pm 7.2 vs. $6.4 \pm 5.0 \%, p=0.002)$ (Figure 5B). As such, comparison between CoreValve and Lotus patients at 6 months showed that the residual total aortic regurgitant fraction was equivalent $(6.4 \pm 5.0$ vs. $5.6 \pm 5.3 \%$ respectively, $p=0.623$ ) (Table 3$)$. Importantly, of the 19 CoreValve patients who were imaged at 6 months, all of the 7 patients with ?moderate AR immediately post-TAVR reduced to only mild AR, while three changed from mild to none/trivial $A R$ There were no Lotus valve patients with $\geq$ moderate $A R$ at any time point (Figure 4).

LV reverse remodelling

There were no significant differences in indexed LV end diastolic volume (LVEDV) $(p=0.379)$, indexed LV mass $(p=0.357)$, LV ejection fraction (LVEF) $(p=0.306)$ or LV mass:volume ratio $(p=0.329)$ between the groups at baseline. A direct comparison of LV morphology and function between the two groups, immediately and at 6 months postTAVR, is summarised in Table 3. Immediately post-TAVR, there was no change in indexed LVEDV or in LVEF in the Lotus group $(p=0.550$ and 0.498$)$ or the CoreValve group ( $p=0.461$ and 0.847 ) respectively. However, a significant reduction in the indexed LV mass occurred following CoreValve TAVR $\left(75.4 \pm 15.0\right.$ vs. $\left.65.8 \pm 13.6 \mathrm{~g} / \mathrm{m}^{2}, \mathrm{p}<0.001\right)$ that was not seen following Lotus $\left(70.8 \pm 25.0\right.$ vs. $\left.69.6 \pm 16.2 \mathrm{~g} / \mathrm{m}^{2}, \mathrm{p}=0.811\right)$. Compared to baseline, the LVEF and indexed LVEDV values at 6 months were unchanged, regardless of the valve type. However, at 6 months, a significant and comparable regression in the indexed LV mass was observed in both TAVR groups (Table 4, Figure 5C and 5D).

\section{DISCUSSION}

This is the first study to use CMR to directly and systematically compare two distinct TAVR designs for aortic valve regurgitation, haemodynamics and impact on LV reverse remodelling over time. The principal findings were as follows: 1. TAVR with the Medtronic CoreValve is associated with a significantly greater quantity of aortic regurgitation 
immediately post-replacement, with over a third of patients having at least moderate AR, compared to none with Lotus valve. 2. AR improves significantly with CoreValve over time, such that none of the patients with $\geq$ moderate AR immediately post-TAVR were left with more than mild AR at 6 months, and there was no longer a difference between valve types in mean aortic regurgitant fraction at 6 months. 3 . The Lotus valve is associated with a significantly higher residual peak gradient than the CoreValve immediately postTAVR. 4. Peak TAVR gradient fell significantly with the Lotus valve over time, with no difference between the valves at 6 months. 5. Despite the differences in aortic valve haemodynamics immediately post-TAVR, left ventricular reverse remodelling at 6 months was equivalent. This is consistent with published studies with longer follow-up that have demonstrated excellent outcomes with both TAVR prostheses ${ }^{15,30},{ }^{30}$

\section{Procedural Success}

In direct comparison, the VARC-defined primary composite outcome of device success was significantly higher in the Lotus group at the immediate post-TAVR time point, driven principally by the absence of $\geq$ moderate aortic regurgitation. The Lotus valve has been compared with the CoreValve previously using echocardiography ${ }^{1}$; however these grading criteria suggested by VARC lack validation post-TAVR ${ }^{31}$. In a recent comparison of $2 \mathrm{D}$ and $3 \mathrm{D}$ echocardiography and CMR, applying VARC metrics post-TAVR, the observer variability in determining AR was superior by CMR ${ }^{12}$. This is the first study to our knowledge to assess device success using CMR, and using VARC criteria suggests superiority of the Lotus valve over CoreValve immediately post deployment. However, device success by the 6 month time point in our study was equivalent.

Aortic Regurgitation following TAVR 
There is growing evidence suggesting a significant association of post-procedural $A R$ with short- and long-term mortality ${ }^{18}, 32,33$ In a meta-analysis of 12,926 patients from 45 studies (a majority using Edwards SAPIEN devices), moderate or more AR was associated with a 2.3-fold increase in 1 year mortality following TAVR ${ }^{34}$. Our study used CMR to study the evolution of AR over time. No significant change was seen in the Lotus group from post-implant to 6 months, in line with REPRISE I ${ }^{15}$ and REPRISE $\|{ }^{16}$. However, we did observe a significant reduction in AR at 6 months following CoreValve replacement. This is consistent with prior echocardiography studies, including the multicentre CoreValve US Pivotal Trial which indicated over $80 \%$ of patients exhibited an improvement of at least 1 grade of regurgitation at 1 year ${ }^{35}$. A recent CMR study did suggest a small increase in AR at 6 months post-TAVR; but this study combined measurements of the CoreValve with those of another TAVR design ${ }^{36}$. Our study is the first to use CMR to demonstrate a significant and clinically important improvement in AR over time; all patients with at least moderate AR immediately post-TAVR having mild or less at 6 months. This may reflect continued outward expansion of the nitinol CoreValve frame ${ }^{35}$, in contrast to the fixed mechanically locked Lotus frame. Interestingly, analysis of the large UK TAVR registry showed $\geq$ moderate AR after CoreValve, in contrast to the SAPIEN valve, was not associated with increased long-term mortality ${ }^{37}$, our finding that $\geq$ moderate AR post-TAVR had resolved at 6 months in all cases may explain these findings.

\section{TAVR Pressure Gradients}

Immediately post-TAVR, we observed a lower residual aortic pressure gradient following CoreValve by an average of $10 \mathrm{mmHg}$ when compared with the Boston Lotus. Previous studies have consistently shown that the CoreValve is associated with low residual 
gradients immediately post valve replacement ${ }^{17,}{ }^{19-21}$, probably due to the supra-annular position of the valve leaflets within the frame, isolating valve function from the surrounding anatomy. Our reported values with the Lotus valve are also comparable to those reported in previous echocardiographic studies ${ }^{16}$. We do not have effective orifice area information as CMR does not permit accurate assessments in this context. Hence the degree of patient - prosthesis mismatch in our study remains unclear and would further clarify whether the pressure gradients represent poor individual sizing or a genuine reflection of TAVR design.

\section{Reverse Remodelling}

A significant finding of this study is the acute regression in LV mass index observed following the CoreValve but not the Lotus. Our group has previously reported acute reverse remodelling following TAVR with an average reduction in $8 \mathrm{~g} / \mathrm{m}^{2}$ seen within the first week ${ }^{38}$. In a sub-study of the PARTNER A trial, a notable portion of patients exhibited mass regression within the first 30 days ${ }^{39}$. These findings may have clinical implications given that early LV mass regression following TAVR is associated with improved diastolic function ${ }^{40}$, lower B-type Natriuretic Peptide levels and reduced readmission to hospital for heart failure ${ }^{39}$. The difference in immediate LV mass regression post TAVR may well be a consequence of the pressure gradients measured; with comparable reverse remodelling observed at 6 months when pressure gradients were similar. Given that the degree of LV mass regression and recovery of diastolic function after TAVR are positive prognostic indicators ${ }^{41}{ }^{42}$ and residual hypertrophy detrimental ${ }^{43}$, further work to validate these findings is merited.

\section{Limitations}


This was a small single-centre non-randomised comparison. Only patients clinically stable enough to participate in the CMR study were included. Not all patients were able to complete the 6 month scan, predominantly due to pacemaker replacement or death, which may have introduced bias, although the final analysed population did not differ from the recruited population in terms of demographics and comorbidities. Our study did include patients in atrial fibrillation (18\% in total) in whom there was potential quantification error. Whilst the VARC-2 criteria include a measure of Patient-Prosthesis Mismatch, defined as absent when the TAVR effective regurgitant orifice area (EOA) is $>0.85 \mathrm{~cm}^{2} / \mathrm{m}^{244}$, we assessed TAVR performance by the original VARC criteria ${ }^{29}$, as our CMR protocol did not include imaging from which TAVR EOA could be ascertained. The VARC criteria are nonetheless widely accepted, forming the basis of a recent randomised clinical trial directly comparing two different TAVR systems ${ }^{20}$.

We used CMR to quantify the total AR seen following TAVR, which is a composite of para-valvular and trans-valvular regurgitation. Total aortic regurgitation following TAVR has been demonstrated as an important marker of mortality ${ }^{45}$ and central trans-valvular regurgitation is usually minor and a physiological feature by virtue of prosthesis design 31. Furthermore, the VARC-2 criteria advocate a combined measurement of "total" aortic regurgitation $(A R)$ reflecting the total regurgitant volume load imposed on the LV ${ }^{44}$. Finally, this study utilised a different grading scale for aortic regurgitant fraction to that advocated by VARC-2, which is based primarily on data from native valve AR measurements. Our values are however entirely consistent with studies focusing on AR specifically after TAVR

\section{CONCLUSIONS}

Immediately post-TAVR, there was significantly less AR but a higher residual peak pressure gradient with the Lotus valve compared to CoreValve. However, at 6 months 
both devices had comparable valve haemodynamics and LV reverse remodelling. 


\section{REFERENCES}

1. Gooley RP, Talman AH, Cameron JD, Lockwood SM, Meredith IT. Comparison of Self-Expanding and Mechanically Expanded Transcatheter Aortic Valve Prostheses. JACC. Cardiovascular interventions. 2015;8:962-971.

2. Pibarot P, Hahn RT, Weissman NJ, Monaghan MJ. Assessment of paravalvular regurgitation following TAVR: a proposal of unifying grading scheme. JACC. Cardiovascular imaging. 2015;8:340-360.

3. Sinning JM, Vasa-Nicotera M, Chin D, et al. Evaluation and management of paravalvular aortic regurgitation after transcatheter aortic valve replacement. Journal of the American College of Cardiology. 2013;62:11-20.

4. Stahli BE, Maier W, Corti R, Luscher TF, Jenni R, Tanner FC. Aortic regurgitation after transcatheter aortic valve implantation: mechanisms and implications. Cardiovascular diagnosis and therapy. 2013;3:15-22.

5. Smith CR, Leon MB, Mack MJ, et al. Transcatheter versus surgical aortic-valve replacement in high-risk patients. The New England journal of medicine. 2011;364:2187-2198.

6. Ussia GP, Barbanti M, Petronio AS, et al. Transcatheter aortic valve implantation: 3-year outcomes of self-expanding CoreValve prosthesis. European heart journal. 2012;33:969-976.

7. Webb JG, Wood DA. Current status of transcatheter aortic valve replacement. Journal of the American College of Cardiology. 2012;60:483-492.

8. Crouch G, Tully PJ, Bennetts J, et al. Quantitative assessment of paravalvular regurgitation following transcatheter aortic valve replacement. Journal of cardiovascular magnetic resonance : official journal of the Society for Cardiovascular Magnetic Resonance. 2015;17:32.

9. Salaun $E$, Jacquier $A$, Theron $A$, et al. Value of CMR in quantification of paravalvular aortic regurgitation after TAVI. European heart journal cardiovascular Imaging. 2015.

10. Sherif MA, Abdel-Wahab M, Beurich HW, et al. Haemodynamic evaluation of aortic regurgitation after transcatheter aortic valve implantation using cardiovascular magnetic resonance. Eurolntervention : journal of EuroPCR in collaboration with the Working Group on Interventional Cardiology of the European Society of Cardiology. 2011;7:57-63.

11. Cawley PJ, Hamilton-Craig C, Owens DS, et al. Prospective comparison of valve regurgitation quantitation by cardiac magnetic resonance imaging and transthoracic echocardiography. Circulation. Cardiovascular imaging. 2013;6:4857.

12. Altiok E, Frick M, Meyer CG, et al. Comparison of two- and three-dimensional transthoracic echocardiography to cardiac magnetic resonance imaging for assessment of paravalvular regurgitation after transcatheter aortic valve implantation. The American journal of cardiology. 2014;113:1859-1866.

13. Ribeiro HB, Le Ven F, Larose $E$, et al. Cardiac magnetic resonance versus transthoracic echocardiography for the assessment and quantification of aortic 
regurgitation in patients undergoing transcatheter aortic valve implantation. Heart. 2014;100:1924-1932.

14. Hartlage GR, Babaliaros VC, Thourani VH, et al. The role of cardiovascular magnetic resonance in stratifying paravalvular leak severity after transcatheter aortic valve replacement: an observational outcome study. Journal of cardiovascular magnetic resonance : official journal of the Society for Cardiovascular Magnetic Resonance. 2014;16:93.

15. Meredith IT, Worthley SG, Whitbourn RJ, et al. Transfemoral aortic valve replacement with the repositionable Lotus Valve System in high surgical risk patients: the REPRISE I study. Eurolntervention : journal of EuroPCR in collaboration with the Working Group on Interventional Cardiology of the European Society of Cardiology. 2014;9:1264-1270.

16. Meredith Am IT, Walters DL, Dumonteil N, et al. Transcatheter aortic valve replacement for severe symptomatic aortic stenosis using a repositionable valve system: 30-day primary endpoint results from the REPRISE II study. Journal of the American College of Cardiology. 2014;64:1339-1348.

17. Adams DH, Popma JJ, Reardon MJ, et al. Transcatheter aortic-valve replacement with a self-expanding prosthesis. The New England journal of medicine. 2014;370:1790-1798.

18. Tamburino $C$, Capodanno D, Ramondo A, et al. Incidence and predictors of early and late mortality after transcatheter aortic valve implantation in 663 patients with severe aortic stenosis. Circulation. 2011;123:299-308.

19. Linke A, Wenaweser P, Gerckens U, et al. Treatment of aortic stenosis with a selfexpanding transcatheter valve: the International Multi-centre ADVANCE Study. European heart journal. 2014;35:2672-2684.

20. Abdel-Wahab M, Mehilli J, Frerker C, et al. Comparison of balloon-expandable vs self-expandable valves in patients undergoing transcatheter aortic valve replacement: the CHOICE randomized clinical trial. Jama. 2014;311:1503-1514.

21. Popma JJ, Adams DH, Reardon MJ, et al. Transcatheter Aortic Valve Replacement Using A Self-Expanding Bioprosthesis in Patients With Severe Aortic Stenosis at Extreme Risk for Surgery. Journal of the American College of Cardiology. 2014.

22. Vahanian A, Alfieri O, Al-Attar N, et al. Transcatheter valve implantation for patients with aortic stenosis: a position statement from the European Association of Cardio-Thoracic Surgery (EACTS) and the European Society of Cardiology (ESC), in collaboration with the European Association of Percutaneous Cardiovascular Interventions (EAPCI). European heart journal. 2008;29:14631470.

23. Piazza N, Grube E, Gerckens U, et al. Procedural and 30-day outcomes following transcatheter aortic valve implantation using the third generation (18 Fr) corevalve revalving system: results from the multicentre, expanded evaluation registry 1 year following CE mark approval. Eurolntervention : journal of EuroPCR in collaboration with the Working Group on Interventional Cardiology of the European Society of Cardiology. 2008;4:242-249. 
24. Meredith IT, Hood KL, Haratani N, Allocco DJ, Dawkins KD. Boston Scientific Lotus valve. Eurolntervention : journal of EuroPCR in collaboration with the Working Group on Interventional Cardiology of the European Society of Cardiology. 2012;8 Suppl Q:Q70-74.

25. Fairbairn TA, Steadman CD, Mather AN, et al. Assessment of valve haemodynamics, reverse ventricular remodelling and myocardial fibrosis following transcatheter aortic valve implantation compared to surgical aortic valve replacement: a cardiovascular magnetic resonance study. Heart. 2013;99:11851191.

26. Dobson LE, Fairbairn TA, Musa TA, et al. Sex-related differences in left ventricular remodeling in severe aortic stenosis and reverse remodeling after aortic valve replacement: A cardiovascular magnetic resonance study. American heart journal. 2016;175:101-111.

27. Gelfand EV, Hughes S, Hauser TH, et al. Severity of mitral and aortic regurgitation as assessed by cardiovascular magnetic resonance: optimizing correlation with Doppler echocardiography. Journal of cardiovascular magnetic resonance : official journal of the Society for Cardiovascular Magnetic Resonance. 2006;8:503-507.

28. Bellenger NG, Davies LC, Francis JM, Coats AJ, Pennell DJ. Reduction in sample size for studies of remodeling in heart failure by the use of cardiovascular magnetic resonance. Journal of cardiovascular magnetic resonance : official journal of the Society for Cardiovascular Magnetic Resonance. 2000;2:271-278.

29. Leon MB, Piazza N, Nikolsky E, et al. Standardized endpoint definitions for transcatheter aortic valve implantation clinical trials: a consensus report from the Valve Academic Research Consortium. European heart journal. 2011;32:205-217.

30. Reardon MJ, Adams DH, Kleiman NS, et al. 2-Year Outcomes in Patients Undergoing Surgical or Self-Expanding Transcatheter Aortic Valve Replacement. Journal of the American College of Cardiology. 2015;66:113-121.

31. Lerakis S, Hayek SS, Douglas PS. Paravalvular aortic leak after transcatheter aortic valve replacement: current knowledge. Circulation. 2013;127:397-407.

32. Kodali SK, Williams MR, Smith CR, et al. Two-year outcomes after transcatheter or surgical aortic-valve replacement. The New England journal of medicine. 2012;366:1686-1695.

33. D'Onofrio A, Facchin M, Besola L, et al. Intermediate Clinical and Hemodynamic Outcomes After Transcatheter Aortic Valve Implantation. The Annals of thoracic surgery. 2015.

34. Athappan G, Patvardhan E, Tuzcu EM, et al. Incidence, predictors, and outcomes of aortic regurgitation after transcatheter aortic valve replacement: meta-analysis and systematic review of literature. Journal of the American College of Cardiology. 2013;61:1585-1595.

35. Oh JK, Little SH, Abdelmoneim SS, et al. Regression of Paravalvular Aortic Regurgitation and Remodeling of Self-Expanding Transcatheter Aortic Valve: An Observation From the CoreValve U.S. Pivotal Trial. JACC. Cardiovascular imaging. 2015;8:1364-1375.

36. Merten $\mathrm{C}$, Beurich HW, Zachow D, et al. Aortic regurgitation and left ventricular remodeling after transcatheter aortic valve implantation: a serial cardiac magnetic 
resonance imaging study. Circulation. Cardiovascular interventions. 2013;6:476483.

37. Dworakowski R, Wendler O, Halliday B, et al. Device-dependent association between paravalvar aortic regurgitation and outcome after TAVI. Heart. 2014;100:1939-1945.

38. Dobson LE, Musa TA, Uddin A, et al. Acute Reverse Remodelling After Transcatheter Aortic Valve Implantation: A Link Between Myocardial Fibrosis and Left Ventricular Mass Regression. The Canadian journal of cardiology. 2016;32:1411-1418.

39. Lindman BR, Stewart WJ, Pibarot $P$, et al. Early regression of severe left ventricular hypertrophy after transcatheter aortic valve replacement is associated with decreased hospitalizations. JACC. Cardiovascular interventions. 2014;7:662673.

40. Vizzardi E, D'Aloia A, Fiorina C, et al. Early regression of left ventricular mass associated with diastolic improvement after transcatheter aortic valve implantation. Journal of the American Society of Echocardiography : official publication of the American Society of Echocardiography. 2012;25:1091-1098.

41. Ali A, Patel A, Ali Z, et al. Enhanced left ventricular mass regression after aortic valve replacement in patients with aortic stenosis is associated with improved long-term survival. The Journal of thoracic and cardiovascular surgery. 2011;142:285-291.

42. Rader F, Sachdev E, Arsanjani R, Siegel RJ. Left ventricular hypertrophy in valvular aortic stenosis: mechanisms and clinical implications. The American journal of medicine. 2015;128:344-352.

43. Beach JM, Mihaljevic T, Rajeswaran J, et al. Ventricular hypertrophy and left atrial dilatation persist and are associated with reduced survival after valve replacement for aortic stenosis. The Journal of thoracic and cardiovascular surgery. 2014;147:362-369 e368.

44. Kappetein AP, Head SJ, Genereux P, et al. Updated standardized endpoint definitions for transcatheter aortic valve implantation: the Valve Academic Research Consortium-2 consensus document. The Journal of thoracic and cardiovascular surgery. 2013;145:6-23.

45. Costopoulos C, Naganuma T, Latib A, Colombo A. Aortic regurgitation after transcatheter aortic valve implantation. Expert review of cardiovascular therapy. 2013;11:1089-1092. 
FIGURE TITLES and LEGENDS

Figure 1: CMR coronal views showing a Medtronic CoreValve (A) and Boston Lotus valve (B).

Figure 2: Study profile.

Figure 3: Aortic Regurgitation classification immediately post-TAVR

Figure 4: Change in TAVR aortic regurgitation over time

Figure 5: Comparison of change over time in valvular and ventricular parameters between the two TAVR designs (mean \pm SE). 